\title{
The Puzzling Stability of Monatomic Gold Wires
}

\author{
J. A. Torres ${ }^{1}$, E. Tosatti ${ }^{1,2,3}$, A. Dal Corso ${ }^{2,3}$, F. Ercolessi ${ }^{2,3}$, \\ J. J. Kohanoff ${ }^{1}$, F. D. Di Tolla ${ }^{2,3}$, and J. M. Soler ${ }^{4}$ \\ 1: Abdus Salam International Centre for Theoretical Physics (ICTP), Trieste, Italy. \\ 2: International School for Advances Studies (SISSA), Trieste, Italy. \\ 3: Istituto Nazionale di Fisica della Materia (INFM), Trieste, Italy. \\ 4: Dep. Física de la Materia Condensada. Univ. Autónoma de Madrid, Spain.
}

(22 December 1998)

\begin{abstract}
We have examined theoretically the spontaneous thinning process of tipsuspended nanowires, and subsequently studied the structure and stability of the monatomic gold wires recently observed by Transmission Electron Microscopy (TEM). The methods used include thermodynamics, classical many-body force simulations, Local Density (LDA) and Generalized Gradient (GGA) electronic structure calculations as well as ab-initio simulations including the two tips. The wire thinning is well explained in terms of a thermodynamic tip suction driving migration of surface atoms from the wire to the tips. For the same reason the monatomic wire becomes progressively stretched. Surprisingly, however, all calculations so far indicate that the stretched monatomic gold wire should be unstable against breaking, contrary to the apparent experimental stability. The possible reasons for the observed stability are discussed.

pacs numbers: 79.60.Jv 61.46.+w 71.24.+q 73.61-r
\end{abstract}

Typeset using REVTEX 
In the last few years the properties of atomic size metallic contacts have attracted great attention both for fundamental and for practical reasons. Experimental formation of these contacts has been achieved using different techniques and in diverse conditions ranging from room temperature under atmospheric pressure [1], to low temperatures in ultrahigh vacuum [2]. The relationship between mechanical structure and electrical properties has been studied by simultaneously measuring load force and conductance [3]. On the theoretical side, the mechanical structure and evolution of a tip-surface mechanical contact has been modelled using classical molecular dynamics (MD) simulations with many body potentials [目, 5, 1], and also by ab-initio MD simulations. [6]. The conductance of these necks has been calculated using free-electron [7], tight binding [8] and ab-intio schemes [9,10]. The relationship between mechanical load and conductance has been studied using different models like free electron [11], tight binding [12] and LDA [13].

Very long tip-suspended nanowires of surprising regularity and stability were found in simulations close to the melting point [4, 114], and subsequently confirmed experimentally [15]. Questions about the structural and thermal behavior of ideally infinite nanowires have recently stirred an upsurge of theoretical [16,17] and experimental interest [19]. New observed structural features of long nanowires include a striking regularity [19]. Both crystalline and noncrystalline morphologies have been predicted [17] and observed [19]. Very recently the exceptional ductility and diffusivity of metallic gold has been put to use in creating the ultimately thin nanowire, consisting of just a single atomic strand [20,21,19]. Direct TEM imaging, [20] in particular, has shown that a thin multi-shell wire suspended between two (110) tips will gradually and spontaneously thin down to smaller and smaller radii, until the two tips are eventually connected by just a 4-atom long monatomic wire. Two of these four $\mathrm{Au}$ atoms stretch out of a well-tapered tips, the remaining two are freely suspended in vacuum in an astonishingly stretched configuration. The stretching magnitude increases with time, the average atomic distances growing from about 3 to about $4 \AA$, yet the wire is not seen to break for many seconds. In different measurements [21],19], the wire was not directly imaged, but conductance plateaus close to and below $G_{0}=2 e^{2} / h$ indicated that the tip-suspended wire must have been monatomic and conducting. For that case, however, no structural information is available. These findings raise several questions, among them:

i) why are the wires thinning down with time, and what is the origin of the stretching force which appears to act on the monatomic wire even in the absence of any mechanical tip withdrawal?

ii) how can we understand the structural stability of such an exceptionally stretched monatomic wire, particularly in terms of its electronic structure?

iii) how can we explain the wire conductance?

While we have not yet addressed iii, the scope of this note is to point out that thermodynamic reasoning readily explains point $i$, whereas preliminary calculations and simulations performed with state-of-the-art methods totally fail to account for the wire stability issue of point $i$. This is all the more surprising since these methods, tuned on the properties of atomic $\mathrm{Au}$, are known to work quite well for bulk and surfaces of metallic $\mathrm{Au}$ [22]. In the following we will address first the thinning. Secondly we will describe our attempts at understanding cohesion and breaking of the infinite wire and of the tip-suspended short wire, and finally speculate on what might be the explanation for the unusual stability. 


\section{WHY DO SUSPENDED WIRES THIN DOWN, AND WHY IS THE MONATOMIC WIRE STRETCHED?}

All wires suspended between two tips appear to thin down steadily with time, on a scale which is visible within seconds. [19,20]. The rate of decrease moreover appears somewhat faster for thinner wires. Once the wire is monatomic, it becomes increasingly stretched with time, even though the tips are fixed relative to one another.

We propose the following simple thermodynamical theory. For a sufficiently thick wire of radius $R$, the free energy per atom is:

$$
\varepsilon(R)=\varepsilon_{0}+2 \gamma / R \rho
$$

where $\varepsilon_{0}$ is the bulk free energy (essentially identical to the cohesive energy), $\gamma$ is the surface free energy per unit area and $\rho$ is the bulk density. Taking one atom from the wire to the tip, the free energy decreases, and in turn this represents an effective suction force exerted by the tips on the wire atoms. In presence of surface diffusion, the wire surface atoms will drift towards the tips, and the wire radius will thus decrease. For a surface diffusion coefficient $D_{s}$ and an Einstein-related surface mobility $\mu_{s}=D_{s} / k_{B} T$, an atom on the wire surface will drift with velocity $v$ towards the tip, and will be absorbed by it in an average time $t \approx L / 4 v$, where $\mathrm{L}$ is the wire length. Due to that, the wire radius will decrease roughly by $\delta R=-1 / 2 \pi R L \rho$. The free energy will first of all decrease by $-2 \gamma / R \rho$ since an atom has left the wire for the (bulk-like) tip. However the wire radius decrease implies an additional free energy cost, all other atoms being left behind in a now thinner wire, amounting to $-\left(2 \gamma / R^{2} \rho\right)\left(\pi R^{2} L \rho-1\right) \delta R$. This cancels one half of the previous free energy decrease (neglecting $\frac{1}{N}$ terms), so that the total free energy change for an atom leaving the wire and going to the tips is, to lowest order in $\frac{1}{R}$,

$$
\Delta E=-\gamma / R \rho
$$

Now the average velocity of surface atom, drifting from the wire middle towards the closest tip, approximately a distance $L / 4$ away, will be $v=\mu_{s}(\Delta E / \Delta x)$, or $v=4 \gamma \mu_{s} / R L \rho$. Assuming all surface particles within an atomic diameter $2 r_{0}$, whose number is $N_{s}=4 \pi L \rho r_{0}\left(R-r_{0}\right)$, to drift in this manner, the radius will decrease with a thinning rate:

$$
\frac{\Delta R}{\Delta t}=\frac{\delta R N_{s}}{L / 4 v}=128 \pi \frac{\gamma \mu_{s} r_{0}^{4}\left(1-r_{0} / R\right)}{3 R L^{2}}
$$

where $\rho=3 / 4 \pi r_{0}^{3}$ has been used. The predicted wire thinning rate calculated from this formula for wires of fixed length and variable radius, with a surface free energy $\gamma_{A u} \sim$ $1.3 \mathrm{~J} / \mathrm{m}^{2}$ and diffusion constant $D_{s}$ ranging from $10^{-5}$ (typical of liquid $\mathrm{Au}$ ) to $10^{-7} \mathrm{~cm}^{2} / \mathrm{s}$, is shown in Fig. 1. As can be seen a typical value for the thinning rate can be in the order of $1 \AA / \mathrm{s}$ for a nanometer thin wire, which seems of the correct magnitude to explain the experimentally observed thinning at room temperature [20]. The thinner and shorter a wire is, the faster it will further thin down, until it gets monatomic. This behavior should be amenable to future experimental verification.

Once the thinning has reduced it to monatomic, the wire can only stretch under the tip suction, until it will eventually break. It is therefore mandatory to estimate the stability of a wire under stretch. 


\section{THE INFINITE MONATOMIC GOLD WIRE}

The electronic structure of an infinite, regular gold monatomic wire has been calculated as a function of the interatomic spacing. The calculation was performed within density functional theory in the LDA, and also in GGA, using the nonlocal ultrasoft pseudopotentials described in reference [23]. A repeated cell geometry was used, with a large inter-wire distance of $10.6 \AA$, and a plane wave expansion with a cutoff energy of 25 Ry for the wavefunctions and 200 Ry for the charge density. The calculated total energy curves versus distance are shown in Fig. 3. They have minima of about $2.2 \mathrm{eV} /$ atom (LDA), or 1.7 $\mathrm{eV} /$ atom (GGA) 24] at an equilibrium distance of about $2.50 \AA$ (LDA) or $2.55 \AA$ (GGA). For reference, with the same pseudopotentials and cut-offs, the calculated cohesive energy for bulk $\mathrm{Au}$ is $4.4 \mathrm{eV}$ (LDA), $3.3 \mathrm{eV}$ (GGA), against an experimental value of $3.9 \mathrm{eV}$. For comparison we also present in Fig. 2 the wire cohesive energy calculated using an

alternative local orbital based LDA and GGA scheme, the SIESTA program 25,26, as well as those given by the empirical glue potential for gold [27]. The wire cohesive energy per atom is $2.8 \mathrm{eV}$ (LDA), $2.1 \mathrm{eV}$ (GGA), 28] and only $0.4 \mathrm{eV}$ (glue). The corresponding bulk cohesive energies provided by the local orbital calculation is $5.4 \mathrm{eV}$ (LDA) $3.9 \mathrm{eV}$ (GGA), $3.9 \mathrm{eV}$ (glue). The four ab-initio calculations are in substantial agreement (we have not attempted a further improvement by extending the basis) while the glue model is seen to severely underbind, and should be discarded for such an ultra-thin wire. [29] Stretching the wire interatomic distance from its equilibrium value to, say, $3.5 \AA$ would cost, according to the local orbital GGA calculation, a stretching energy of about $4.0 \pm 0.5 \mathrm{eV}$, nearly exactly compensating the energy gain corresponing to one gold atom sucked by one of the tips, namely the bulk cohesive energy, which is $3.9 \mathrm{eV}$. This would seem a very reasonable balance, supporting the concept that tip suction is indeed responsible for the observed wire stretching.

However, this reasoning is not further supported by the electronic structure calculations where we find that even a relatively modest stretching endangers the wire stability. Using for simplicity a four atom cell with periodic boundary conditions, we tested for longitudinal stability by calculating total energy changes when one interatomic spacing is extended from $a$ to $a^{\prime}$, while forcing the three remaining interatomic distances to shrink so as to keep a

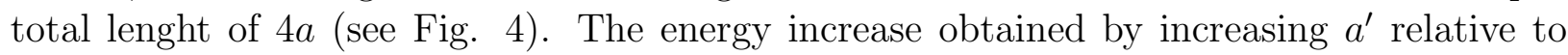
$a$ represents the energy barrier which prevents the wire from longitudinal breaking. To our surprise, we found (Fig. 3) that the barrier is positive at the equilibrium spacing of $2.55 \AA$ and just above, but disappears very quickly when the wire is stretched, making it unstable below $3 \AA$. 30] This result is puzzling, in view of the exceptional stability observed experimentally. However, there is still a distinct possibility that the real 4-atom wire hanging between well-tapered tips could in many ways behave differently from the tip-less wire just studied. We thus decided to investigate further a more realistic case.

\section{SHORT MONATOMIC WIRE BETWEEN (110) TIPS: AB-INITIO SIMULATION}

For the first-principles MD relaxation, we generated a starting configuration in the following manner. We started with an FCC bulk-ordered cubic-shaped piece of 240 atoms. 
Keeping both extreme layers rigid, we separated them at constant velocity along the (110) direction and simulated the evolution of the neck using glue model MD (so long as atomic coordination not too poor, the glue model provides quite reasonable interatomic forces for Au.) The stretching velocity was $\approx 0.14 \mathrm{~m} \AA /$ fs, and the temperature was kept very low. $\mathrm{A}$ neck formed and broke. After neck breaking we stopped the simulation and inserted three additional atoms in a row, forming a wire bridging the opened gap. Finally, we relaxed the new configuration, and the interatomic spacing of the relaxed wire reached about $2.9 \AA$. We further pulled the tips, stretching the monatomic wire up to the very moment before it would break and stopped there, finally relaxing and checking for stability with glue forces. The 33 central atoms of this last configuration, shown in Fig. 1 form our initial configuration, with two tapered tips and a 4 -atom wire with an interatomic spacing of about $3.5 \AA$.

Starting with this configuration, we calculated the electronic structure and the forces on all 33 atoms using the local basis LDA [31]. With these forces we conducted a short ab initio MD relaxation of 40 steps of $5 \mathrm{fs}$ each plus 60 steps of $7 \mathrm{fs}$ each. The resulting ab-initio evolution after 100, 200, 340, 480 and 620 fs is also shown in Fig. 4. We see that interatomic distances in the wire change rapidly. However, while most of them shorten, especially close to the tip, one distance is growing with time, and the wire is clearly breaking. It appears therefore that within this approximation, the short wire hanging from the tips is unstable, very much as the infinite wire was.

\section{DISCUSSION}

We believe we have understood the thinning and stretching of wires between tips, and provided a thinning rate dependence upon the wire length and radius that can be checked experimentally. The exceptional stability of the stretched monatomic wire remains instead mysterious since state-of-the-art calculations predict that it should break by far. While independent measurements would clearly be desirable in order to confirm the large spacings observed in reference [20], we can presently only speculate on the possible reasons why our theory might be in error on this problem.

A first possibility is that electron correlations, weak in bulk $\mathrm{Au}$, could become strong in severely stretched wires, owing to the exceedingly poor coordination, and to the large interparticle distance. In particular, typical one dimensional instabilities, involving antiferromagnetism or dimerization could play a role. Of them, dimerization is allowed in our simulation, but magnetism is not. While this is clearly an avenue to be explored, it seems at this stage unlikely that it could alter the energetics very drastically. All the same, if the stretched wire turned magnetic (and presumably also insulating), the corresponding small energy decrease might be sufficient to generate a small change of curvature and therefore a small barrier against breaking.

A second factor which we neglected throughout is long-range correlations, and particularly van der Waals forces. Van der Waals attraction is known to be strong especially between blunt tips [32]. This attraction could be enhanced by the presence of a conducting wire joining the two tips, thus providing the missing barrier. This effect is absent in LDA/GGA which does not include long-range dispersion forces.

An alternative possibility is that the TEM electron beam could be influencing, by heating or charging, the wire, and causing its anomalous behavior. However both tips are electrically 
grounded, and it is further estimated [33] that only about one electron per second collides elastically with the wire, the inelastic cross section being even smaller.

Clearly the problem remains open, and more experimental and theoretical work will be needed to solve it.

We are grateful to Prof. K. Takayanagi and his group for enlightening discussions. We acknowledge financial support from INFM/G (Project "Nanowires"), INFM/F (PRA LOTUS), by MURST through COFIN97, and Spain's DGES, project PB95-0202. Work by J.A.T. was carried out under TMR grant ERBFMBICT972563. 


\section{REFERENCES}

[1] J. I. Pascual, J. Méndez, J. Gómez-Herrero, A. M. Baró, N. García, U. Landman, W. D. Luedtke, E. N. Bogachek, and H. P. Cheng, Science 267, 1793 (1995).

[2] J.M. Krans, J.M. van Ruitenbeek, V.V. Fisun, I.K. Yanson, and L.J. de Jongh, Nature 375, 6534 (1995).

[3] G. Rubio, N. Agrait, and S. Vieira, Phys. Rev. Lett. 76, 2302 (1996).

[4] O. Tomagnini, F. Ercolessi, and E. Tosatti, Surf. Sci. 287/288, 1041 (1993).

[5] M.R. Soerensen, M. Brandbyge, and K.W. Jacobsen, Phys. Rev. B 57, 3283 (1998) and references therein.

[6] R. N. Barnet and U. Landman, Nature 387, 788 (1997).

[7] J.A. Torres, J.I. Pascual, and J.J. Sáenz, Phys. Rev. B 49, 16581 (1994); A. GarcíaMartín, J. A. Torres, and J. J. Sáenz, Phys. Rev. B. 54, 13448 (1996); J.I. Pascual, J.A. Torres, and J.J. Sáenz, Phys. Rev. B 55, R16029 (1997).

[8] C. Sirvent, J.G. Rodrigo, S. Vieira, L. Jurczyszyn, N. Mingo, and F. Flores, Phys. Rev. B 53, 16086 (1996).

[9] N.D. Lang, Phys. Rev. B 52, 5335 (1995).

[10] F. Di Tolla et al., unpublished.

[11] J. A. Torres and J. J. Sáenz, Phys. Rev. Lett. 77, 2245 (1996).

[12] T.N. Todorov and A.P. Sutton, Phys. Rev. Lett. 70, 2138 (1993).

[13] D. Sanchez and J.M. Soler, Phys. Rev. Lett. 79, 4198 (1997).

[14] O. Tomagnini, F. Ercolessi, and E. Tosatti, unpublished.

[15] L. Kuipers and J.W.M. Frenken, Phys. Rev. Lett. 70, 3907 (1993).

[16] O. Gulseren, F. Ercolessi and E. Tosatti, Phys. Rev. B 51, 7377 (1995).

[17] O. Gulseren, F. Ercolessi and E. Tosatti, Phys. Rev. Lett. 80, 3775 (1998).

[18] H. Ohnishi, Y. Kondo, K. Takayanagi, to be published.

[19] H. Ohnishi, Y. Kondo, and K. Takayanagi, Phys. Rev. Lett. 79, 3455 (1997).

[20] H. Ohnishi, Y. Kondo, and K. Takayanagi, Nature 395, 780 (1998).

[21] A.I. Yanson, G. Rubio, H.E. van der Brom, N. Agrait, and J.M. van Ruitenbeek, Nature 395, 783 (1998).

[22] N. Takeuchi, C. T. Chan, and K. M. Ho, Phys. Rev. Lett. 63, 1273 (1989).

[23] A. Dal Corso, A. Pasquarello, and A. Baldereschi, Phys. Rev. B 56, R11369 (1997).

[24] J.P. Perdew, in Electronic Structure of solids '91, edited by P. Ziesche and H. Eschrig (Akademic-Verlag, Berlin 1991).

[25] D. Sanchez Portal, P. Ordejon, E. Artacho, and J. M. Soler, Int. Journal of Quantum Chemistry 65, 453 (1997).

[26] A nonlocal norm conserving scalar relativistic Troullier-Martins pseudopotential [N. Troullier and J.L. Martins, Phys. Rev. B 43, 1993 (1991)] was incorporated so that only eleven valence electrons per gold atoms were considered. Tests of the pseudopotential as well as the basis set were realized on gold dimer and bulk gold. Specifically 150 Ry in the energy cutoff of the finite real-space grid and double-z $s$, single-z $p$, and double-z $d$ orbitals were used to obtain small relative errors (compared to experimental values) in the dimer equilibrium distance $(0.8 \%)$ with checks up to 250 Ry and a polarized double-z basis.

[27] F. Ercolessi, M. Parrinello and E. Tosatti, Philos. Mag. A. 58, 213 (1988).

[28] J.P. Perdew, K. Burke, and M. Ernzerhof, Phys. Rev. Lett. 77, 3865 (1996). 
[29] There is in reality a deeper minimum $(1.2 \mathrm{eV})$ for the glue model at $1.73 \AA$, but it is clearly unphysical.

[30] A reversal of concavity, from positive to negative in the energy vs. distance curve of Fig. 2 occurs in fact at $\sim 2.8 \AA$. It can be seen as giving rise to the instability, e.g. via negative force constants.

[31] The same basis was used as in the infinite wire case [26]. This time the energy cutoff of the finite real-space grid was 100 Ry.

[32] E. Tosatti, in Highlights in condensed matter physics and future prospects Ed. by L. Esaki (Plenum, New York, 1991) p. 631.

[33] K. Takayanagi private communication. 


\section{FIGURES}

FIG. 1. Thinning rate of tip suspended $20 \AA$ long gold wires, from Eq. 2, calculated for different surface diffusion constants.

FIG. 2. Cohesive energy per atom for an infinite monatomic Au wire.

FIG. 3. Energy variation upon attempted wire breaking. The wire is not stable when the interatomic spacing is stretched above $2.8 \AA$.

FIG. 4. Snapshots of the ab-initio MD relaxation for increasing time. The spontaneous breaking of the wire is evident. 


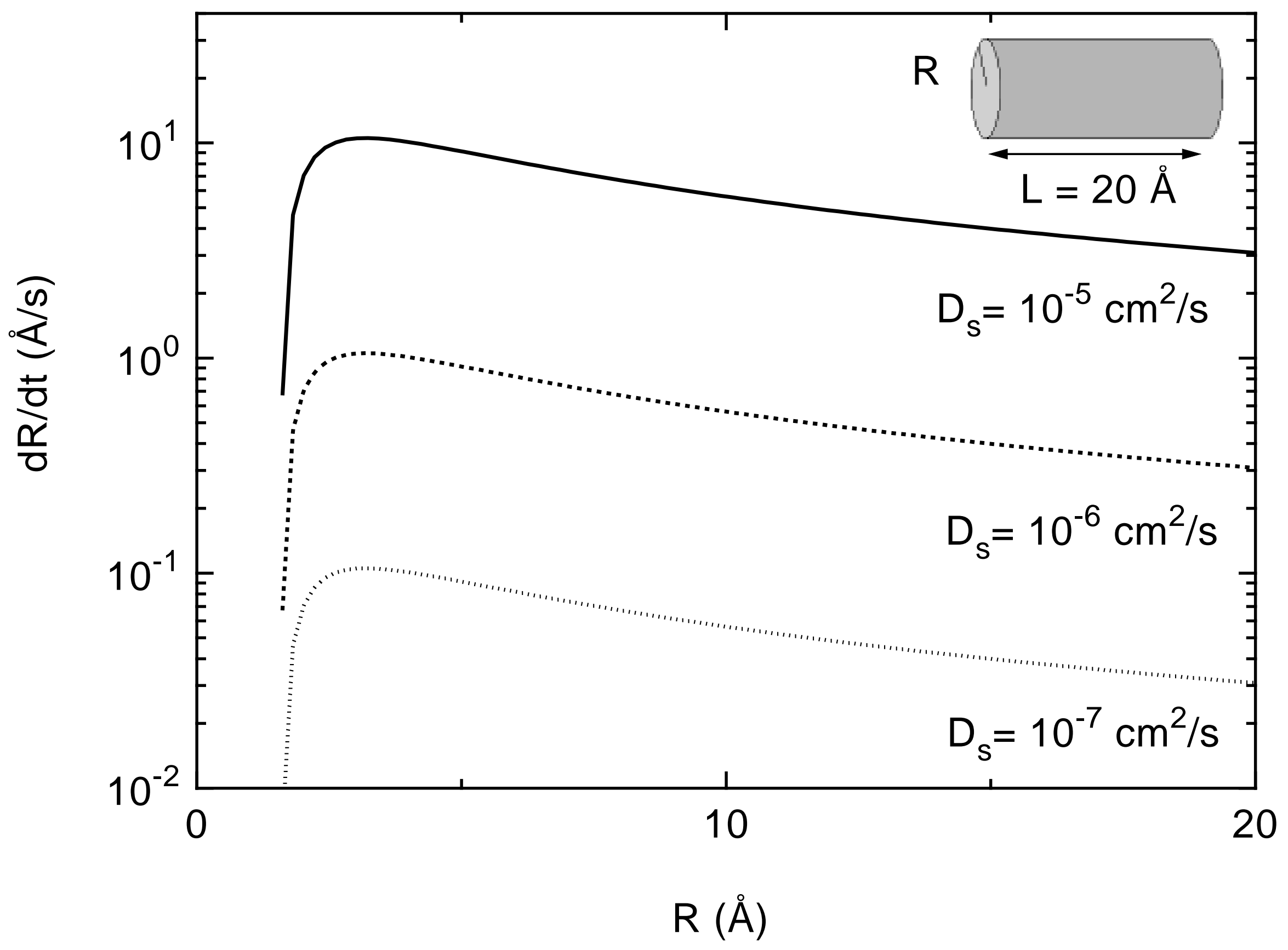




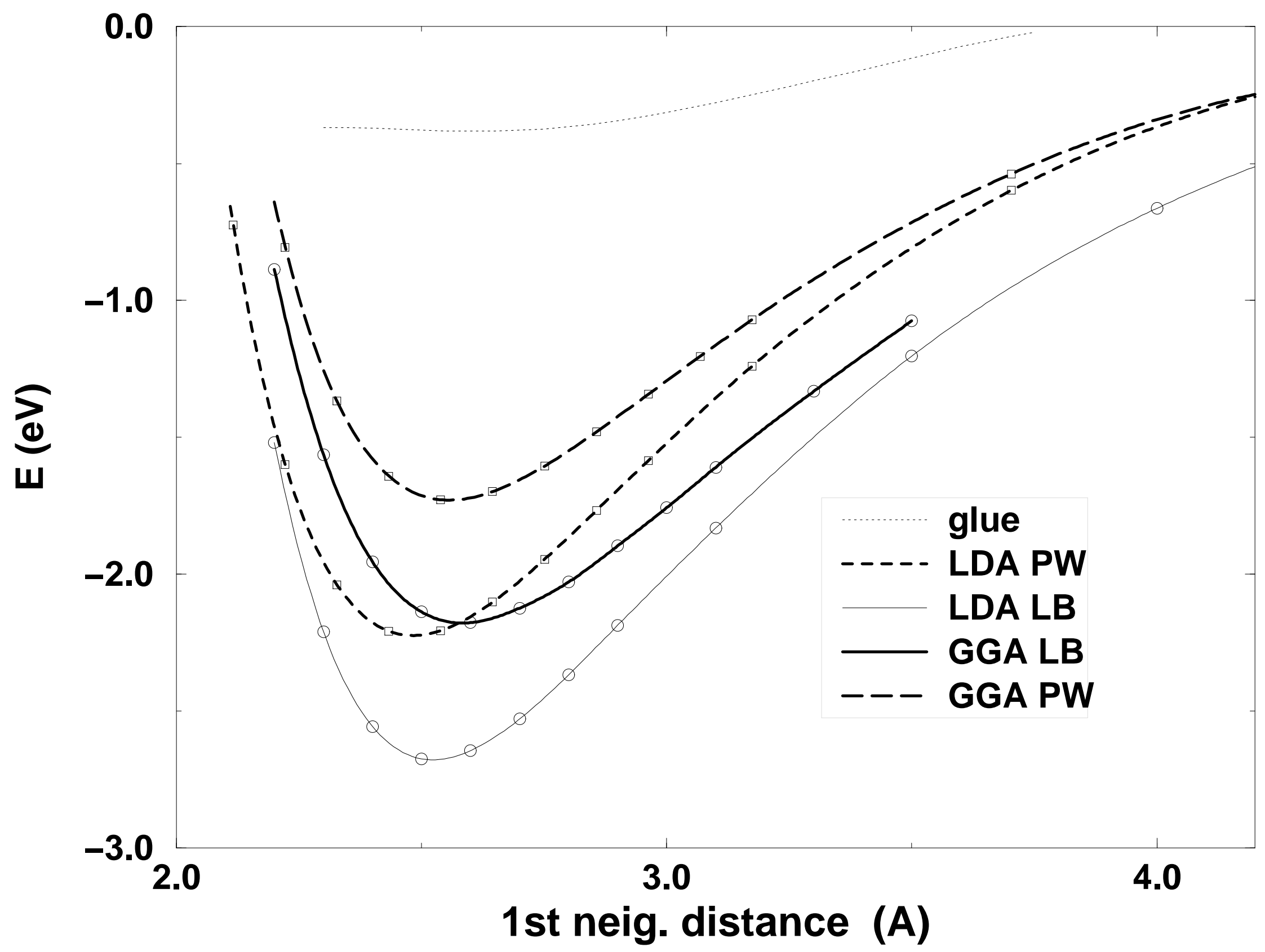




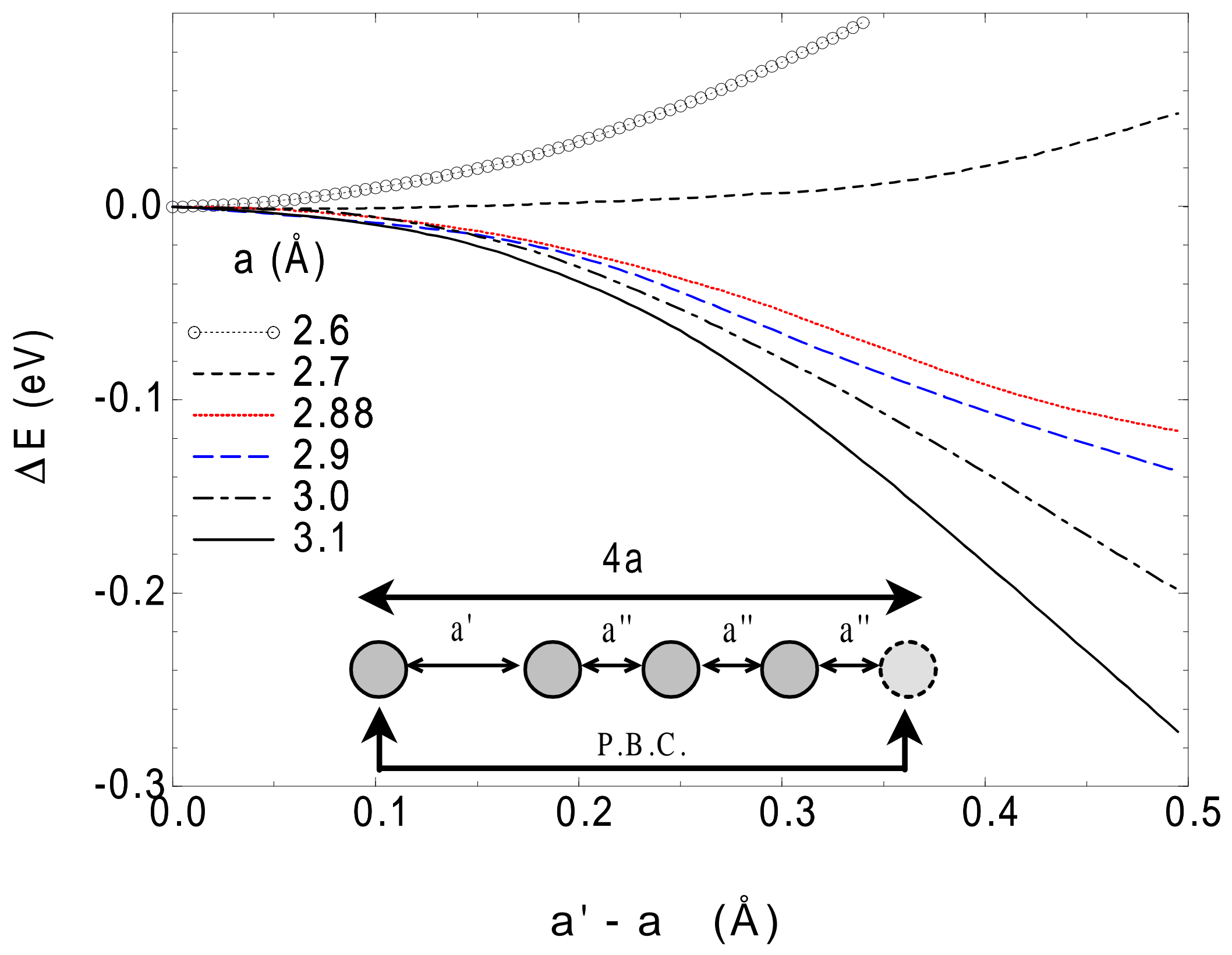




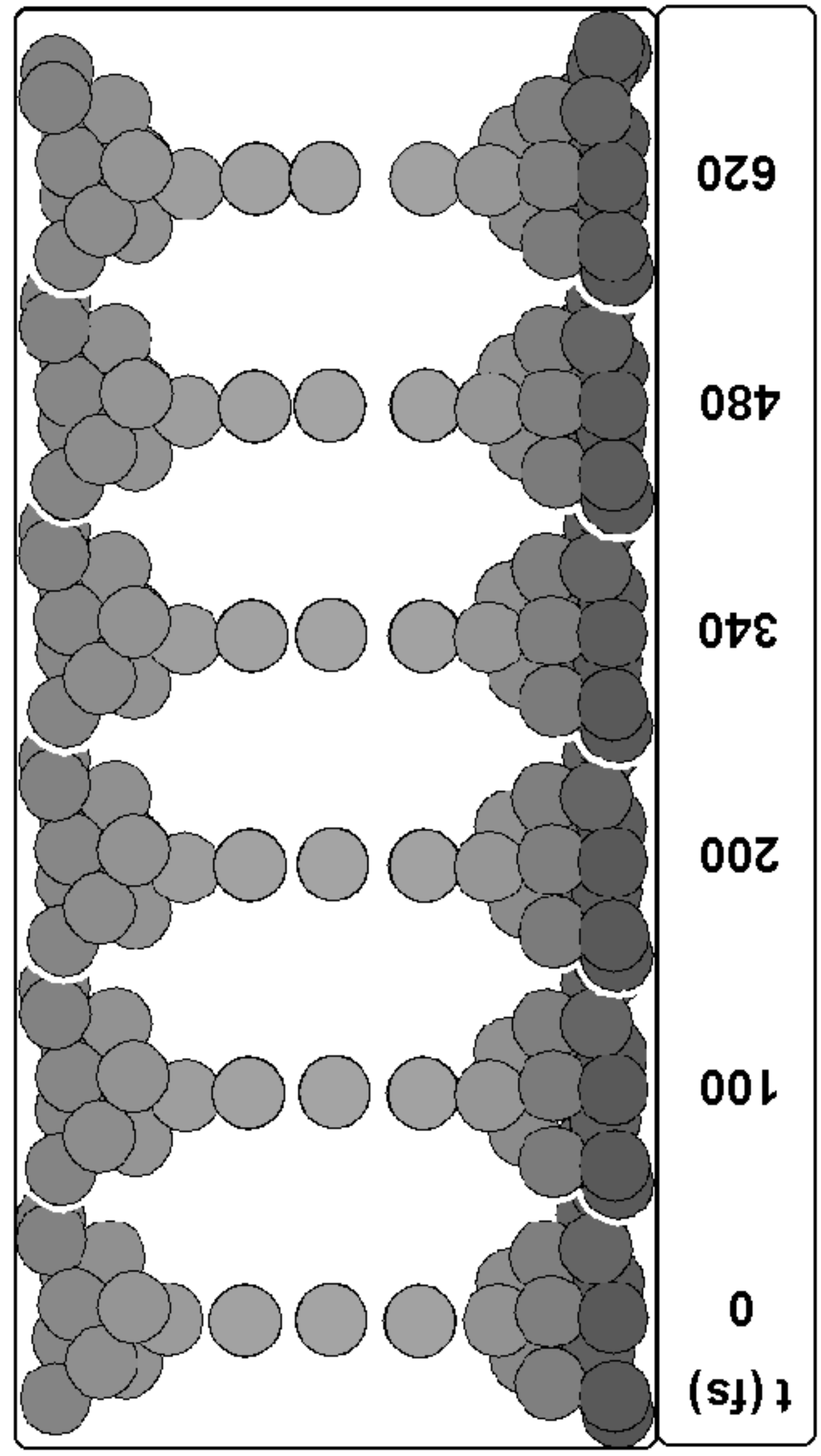

